\title{
The Second Largest Prime Factor of an Odd Perfect Number
}

\author{
By Carl Pomerance
}

\begin{abstract}
Recently Hagis and McDaniel have studied the largest prime factor of an odd perfect number. Using their results, we begin the study here of the second largest prime factor. We show it is at least 139 . We apply this result to show that aAy odd perfect number not divisible by eight distinct primes must be divisible by 5 or 7 .
\end{abstract}

1. Introduction. Suppose $n=p_{1}^{a_{1}} p_{2}^{a_{2}} \cdots p_{t}^{a_{t}}$ is an odd perfect number (opn) where $a_{1}, a_{2}, \ldots, a_{t}$ are positive integers and $p_{1}>p_{2}>\cdots>p_{t}$ are primes. In [7], Kanold proved that $p_{1} \geqslant 61$. Recently, Hagis and McDaniel [5] , [6] have succeeded in showing that

$$
p_{1} \geqslant 100129 \text {. }
$$

It is the purpose of this paper to study the second largest prime factor of an opn. In Section 2 we develop a method of attack. In Section 3 we prove that

$$
p_{2} \geqslant 139 \text {. }
$$

The proof of (2) makes only marginal direct use of computers (we used some computer factorizations in the construction of Table 3). But the proof of (2) does depend strongly on (1), and (1) could not have been accomplished without electronic assistance.

In Section 4 we illustrate the value of the seemingly weak (2). Indeed, in Pomerance [9] and Robbins [10] it is shown that any opn is divisible by at least 7 distinct primes. Sylvester [11] showed that if an opn $n$ is divisible by precisely 7 distinct primes, then $3 \mid n$. In Section 4, using (1) and (2), we prove that if an opn $n$ is divisible by precisely 7 distinct primes, then either $5 \mid n$ or $7 \mid n$. This result would be very difficult to establish without the use of (2).

Before we proceed, it should be pointed out that there is an effective (but not practical) procedure for deciding the following:

Problem. Given any $k, N$, either find an opn with $k$ th largest prime factor $p_{k}<$ $N$, or prove no such opn exists.

Indeed, Dickson [2] and Gradštein [4] proved that for any given $m$ there are only finitely many opn's divisible by at most $m$ distinct primes, and it is clear, at least from Dickson's proof, that these opn's are effectively computable. Hence, to resolve the above problem, one need only examine the finite set of opn's divisible by at most $k+\pi(N)-2$ distinct primes (where $\pi(N)$ denotes the number of primes less than $N$ ). Indeed, if the $k$ th largest prime $p_{k}$ of the opn $n$ satisfies $p_{k}<N$, then $n$ is divisible by

Received July 10, 1974.

AMS (MOS) subject classifications (1970). Primary $10 \mathrm{~A} 20$.

Copyright $\odot 1975$, American Mathematical Society 
at most $k-1$ distinct primes not less than $N$. Together with the $\pi(N)-1$ odd primes less than $N$; we see that $n$ is divisible by at most $k+\pi(N)-2$ distinct primes.

Using this procedure to prove (2), one would "merely" have to enumerate the set of opn's divisible by at most 33 distinct primes. However, this would be an enormous undertaking and hardly practical. Since we do know that every opn is divisible by at least 7 distinct primes, this method gives the result $p_{2} \geqslant 17$. Using the additional fact that no opn is divisible by $3 \cdot 5 \cdot 7$ (Sylvester [12]), we can get $p_{2} \geqslant 19$.

By the above discussion, for any $N$ there are at most a finite number of opn's with second largest prime factor $p_{2}<N$. We denote this set by $P(N)$. Hence, given any prime $p$, the set $E(p, N)=\left\{a>0: p^{a} \| n\right.$ for some $\left.n \in P(N)\right\}$ is finite (we write $x \| y$ if $x \mid y$ and $(x, y / x)=1)$. The main goal of Section 2 is to develop procedures for proving $E(p, N)=\varnothing$ or perhaps $E(p, N) \subset S$ where $S$ is some small, explicitly determined set of positive integers.

I wish to acknowledge the expert assistance of David E. Penney on the computer work used for constructing Table 3.

2. Notation, Preliminaries, and a Theorem. If $p$ is a prime and $m$ is a natural number, we write $v_{p}(m)$ for the exponent (possibly 0 ) on $p$ in the prime factorization of $m$. If $p / m$, we write $\operatorname{ord}_{p}(m)$ for the least positive integer $h$ for which $p \mid\left(m^{h}-1\right)$. We write

$$
\begin{array}{ll}
a_{p}(m)=0, & \text { if } \operatorname{ord}_{p}(m)=1 ; \\
a_{p}(m)=v_{p}\left(m^{h}-1\right), & \text { if } h=\operatorname{ord}_{p}(m)>1 .
\end{array}
$$

LEMMA 1. If $p$ is a prime and $m=q^{c}>1$ where $q, c$ are natural numbers, then $a_{p}(q) \leqslant a_{p}(m)+v_{p}(m-1)$.

We denote by $\sigma(m)$ the sum of the positive divisors of $m$. We note that if $q$ is a prime, then $\sigma\left(q^{c}\right)=\left(q^{c+1}-1\right) /(q-1)$ for any natural number $c$. The following lemma is a corollary of Theorems 94 and 95 in Nagell [8].

LeмmA 2. If $p, q$ are odd primes and $a, c$ are natural numbers, then $p^{a} \| \sigma\left(q^{c}\right)$ if and only if $\operatorname{ord}_{p}(q) \mid(c+1)$ and $v_{p}(c+1)=a-a_{p}(q)$.

LEMмA 3. If $p, q$ are odd primes, $a, b$ are natural numbers, and $\sigma\left(p^{a}\right)=q^{b}$, then $a_{p}(q)=0$ or 1 .

Proof. We have $q^{b}-1=p+p^{2}+\cdots+p^{a}$, so that $p \|\left(q^{b}-1\right)$. Let $h=$ $\operatorname{ord}_{p}(q)$. Then $h \mid b$ and $p \|\left(q^{h}-1\right)$.

We state now a result found often in the literature; it is originally due to Bang [1]: Given arbitrary integers $a \geqslant 2, b \geqslant 2$, there is a prime $p$ with ord $p(a)=b$, unless (i) $a=2$ and $b=6$, or (ii) $a=2^{k}-1$ for some $k$ and $b=2$.

LEMMA 4. If $q$ is an odd prime, $c$ is a natural number, and $4 / \sigma\left(q^{c}\right)$, then for each divisor $d>1$ of $c+1$, there is a prime $p \mid \sigma\left(q^{c}\right)$ with $\operatorname{ord}_{p}(q)=d$.

Proof. We apply the theorem quoted above to the natural numbers $q, d$, noting that $q \neq 2$ so (i) does not apply, and since $4 / \sigma\left(q^{c}\right)$ we have $c+1$ odd if $q=2^{k}-1$ for some $k$, so (ii) does not apply. Hence there is a prime $p$ with $\operatorname{ord}_{p}(q)=d$. Also, $p\left|\left(q^{d}-1\right) /(q-1)\right|\left(q^{c+1}-1\right) /(q-1)=\sigma\left(q^{c}\right)$.

Suppose now $N<100129$ and $p$ is an odd prime. We denote by $A(p, N)$ the set 
of all $q^{c}, q$ an odd prime, $c$ a natural number, with

(i) $q<N$;

(ii) $p \mid \sigma\left(q^{c}\right)$;

(iii) $4 / \sigma\left(q^{c}\right)$;

(iv) if $c^{\prime}<c$, then $v_{p}\left(\sigma\left(q^{c^{\prime}}\right)\right)<v_{p}\left(\sigma\left(q^{c}\right)\right)$; and either

(v) every prime factor of $\sigma\left(q^{c}\right)$ is less than $N$; or

(vi) $\sigma\left(q^{c}\right)$ has precisely one prime factor $r \geqslant N$ and $r \geqslant 100129$

We remark that given any $q, p, N$, there are at most finitely many $c$ with $q^{c} \in A(p, N)$. Indeed, Lemma 2 and condition (iv) imply that if $c+1 \neq \operatorname{ord}_{p}(q)$, then $p \mid(c+1)$. But if $c+1=p m$ where $m \geqslant N$, then Lemma 4 implies there are primes $r_{1}, r_{2}$ with $r_{1} r_{2} \mid \sigma\left(q^{c}\right), \operatorname{ord}_{r_{1}}(q)=m$, and $\operatorname{ord}_{r_{2}}(q)=p m$. Then $r_{1} \geqslant m+1>N, r_{2} \geqslant p m+1$ $>N$, so that neither (v) nor (vi) is satisfied. We conclude that the set $A(p, N)$ is effectively computable.

We now define several subsets of $A(p, N)$. Let $A_{1}(p, N)$ denote the subset of those $q^{c}$ for which $2 \mid \sigma\left(q^{c}\right)$; let $A_{2}(p, N)$ denote the subset of those $q^{c}$ for which $2 \gamma \sigma\left(q^{c}\right)$ and (v) holds; and let $A_{3}(p, N)$ denote the subset of those $q^{c}$ for which $2 \gamma_{\sigma}\left(q^{c}\right)$ and (vi) holds. Let

$$
\begin{aligned}
& a_{1}(p, N)=\max \left\{v_{p}\left(\sigma\left(q^{c}\right)\right): q^{c} \in A_{1}(p, N)\right\}, \\
& a_{2}(p, N)=a_{1}(p, N)+\sum_{q} \max _{c}\left\{v_{p}\left(\sigma\left(q^{c}\right)\right): q^{c} \in A_{2}(p, N)\right\}, \\
& a_{3}(p, N)=a_{1}(p, N)+\sum_{q} \max _{c}\left\{v_{p}\left(\sigma\left(q^{c}\right)\right): q^{c} \in A_{2}(p, N) \cup A_{3}(p, N)\right\} .
\end{aligned}
$$

Let

$$
\begin{aligned}
& Q(p, N)=\left\{r \geqslant 100129: r \text { prime, } r \mid \sigma\left(q^{c}\right) \text { for some } q^{c} \in A_{3}(p, N)\right\} \\
& b(p, N)=\max \left\{v_{p}(q-1): q \text { prime, } q<N\right\}
\end{aligned}
$$

We recall now the definitions of $P(N)$ and $E(p, N)$ from Section 1 .

Lemma 5. Suppose $n \in P(N)$ where $N<100129, p_{1}$ is the largest prime factor of $n, p$ is any prime factor of $n, p_{1}^{a_{1}} \| n$, and $p^{a} \| n$. Then $v_{p}\left(\sigma\left(p_{1}^{a_{1}}\right)\right) \geqslant a-a_{3}(p, N)$. If $p_{1} \notin Q(p, N)$, then $v_{p}\left(\sigma\left(p_{1}^{a_{1}}\right)\right) \geqslant a-a_{2}(p, N)$.

Proof. Let the prime factorization of $n$ be written $p_{1}^{a_{1}} p_{2}^{a_{2}} \cdots p_{t}^{a_{t}}$ where $p_{1} \geqslant$ $100129>N>p_{2}>\cdots>p_{t}$. Now $\sigma(n)=2 n$, so $a=\Sigma_{i=1}^{t} v_{p}\left(\sigma\left(p_{i}^{a_{i}}\right)\right)$. But $\Sigma_{i=2}^{t} v_{p}\left(\sigma\left(p_{i}^{a_{i}}\right)\right) \leqslant a_{3}(p, N)$, and if $p_{1} \notin Q(p, N)$, then $\Sigma_{i=2}^{t} v_{p}\left(\sigma\left(p_{i}^{a_{i}}\right)\right) \leqslant a_{2}(p, N)$.

THEOREM 1. Let $N<100129$ and let $p$ denote an odd prime. Each of the following conditions implies $a \notin E(p, N)$ :

(i) $4 \mid \sigma\left(p^{a}\right)$;

(ii) there is a prime $q \mid \sigma\left(p^{a}\right)$ with $N \leqslant q<100129$;

(iii) there are primes $q_{1}, q_{2}$ with $q_{1} q_{2} \mid \sigma\left(p^{a}\right)$ and $q_{1}>q_{2} \geqslant N$;

(iv) $\sigma\left(p^{a}\right)$ is divisible by a prime $q \geqslant N$ and $a>a_{3}(p, N)+b(p, N)+a_{p}(q)$;

(v) $\sigma\left(p^{a}\right)$ is divisible by a prime $q \geqslant N, q \notin Q(p, N)$, and $a>a_{2}(p, N)+b(p, N)$ $+a_{p}(q)$

(vi) every prime divisor of $\sigma\left(p^{a}\right)$ is at least $N$ and $a>a_{3}(p, N)+b(p, N)+1$; 
(vii) $\sigma\left(p^{a}\right)=m_{1} m_{2}$ where every prime divisor of $m_{1}$ is less than $N$, every prime divisor of $m_{2}$ is at least $N, m_{2}>1$, and $a>a_{3}(p, N)+b(p, N)+a_{p}\left(m_{2}\right)+v_{p}\left(m_{2}-1\right)$;

(viii) $p<N, a+1$ is prime, and $a+1 \geqslant \max \left\{1 / 2(N-1), a_{3}(p, N)+b(p, N)+\right.$ $3\}$;

(ix) $p^{a^{\prime}}$ satisfies one of the above and $\left(a^{\prime}+1\right) \mid(a+1)$.

Proof. Note that (i), (ii), and (iii) are obvious.

Suppose (iv) holds and suppose $n \in P(N)$ with $p^{a} \| n$. Then $q=p_{1}$, the largest prime factor of $n$. Say $p_{1}^{a_{1}} \| n$. Lemma 5 implies $v_{p}\left(\sigma\left(p_{1}^{a_{1}}\right)\right) \geqslant a-a_{3}(p, N)>b(p, N)$ $+a_{p}\left(p_{1}\right)$. Hence Lemma 2 implies $p^{1+b(p, N)} \mid\left(a_{1}+1\right)$. By Lemma 4, there is a prime $r \mid \sigma\left(p_{1}^{a_{1}}\right)$ with $\operatorname{ord}_{r}\left(p_{1}\right)=p^{1+b(p, N)}$, so that $v_{p}(r-1) \geqslant 1+b(p, N)$. Then $r \geqslant N$. But $r \neq p_{1}$, contradicting $n \in P(N)$. Hence $a \notin E(p, N)$.

To show that (v) implies $a \notin E(p, N)$ we proceed as with the proof of (iv), except that we note the condition $p_{1}=q \notin Q(p, N)$ implies by Lemma 5 that $v_{p}\left(\sigma\left(p_{1}^{a} 1\right)\right)$ $\geqslant a-a_{2}(p, N)$.

Now assume (vi) holds. Then we may assume $\sigma\left(p^{a}\right)=q^{b}$ where $q$ is a prime and $q \geqslant N$. Lemma 3 implies $a_{p}(q)=0$ or 1 . Hence $a \notin E(p, N)$ by (iv).

If (vii) holds, we may assume $m_{2}=q^{b}$ where $q$ is a prime and $q \geqslant N$. Then Lemma 1 and (iv) imply $a \notin E(p, N)$.

Suppose (viii) holds. Since $a+1$ is prime, if $q$ is a prime divisor of $\sigma\left(p^{a}\right)$, then $\operatorname{ord}_{q}(p)=a+1$ or 1 by Lemma 2 . In the former case, $q \equiv 1(\bmod a+1)$, so $q \geqslant$ $2(a+1)+1 \geqslant N($ since $a+1 \geqslant 3$ and $a+1 \geqslant 1 / 2(N-1))$. Suppose $\operatorname{ord}_{q}(p)=1$, so that $p \equiv 1(\bmod q)$. Then $\sigma\left(p^{a}\right)=1+p+\cdots+p^{a} \equiv a+1(\bmod q)$; so that $a+$ $1=q$. Hence $p \geqslant 2(a+1)+1 \geqslant N$, a contradiction. Thus every prime divisor of $\sigma\left(p^{a}\right)$ is at least $N$, and since $a>a_{3}(p, N)+b(p, N)+1$, (vi) implies $a \notin E(p, N)$.

Finally, suppose (ix) holds. Since $\sigma\left(p^{a}\right) \mid \sigma\left(p^{a}\right)$, we have $a \notin E(p, N)$ due to our above proofs for (i)-(viii).

Remark. We note that Lemma 4 together with (iii), (viii), and (ix) of Theorem 1 imply that if $a \in E(p, N)$ and $p<N$, then every prime divisor $r$ of $a+1$ satisfies $r<$ $\max \left\{1 / 2(N-1), a_{3}(p, N)+b(p, N)+3\right\}$ and $v_{r}(a+1) \leqslant b(r, N)+1$. Hence Theorem 1 provides an effective means for examining the finite set $E(p, N)$.

3. The Proof of (2). In Section 2 we remarked that the sets $A(p, N)$ (and hence the subsidiary notions $A_{1}(p, N), A_{2}(p, N), A_{3}(p, N), Q(p, N), a_{1}(p, N), a_{2}(p, N)$, and $\left.a_{3}(p, N)\right)$ are effectively computable. We have performed these computations for $N=$ 139 and $p=3,7,11,13,19,31,61,97$, and 127 . The fruits of this labor may be found in Tables 1, 2, and 3. Also, $b(p, 139)$ for the above $p$ may be found in Table 1. Making use of this numerical information and Theorem 1, we are able to conclude:

$$
\begin{array}{rlrl}
E(3,139) & \subset\{2,4\}, & & E(31,139)=\varnothing, \\
E(7,139) & \subset\{2\}, & E(61,139) & \subset\{1,2\}, \\
E(11,139) & \subset\{2\}, & E(97,139) & \subset\{1\}, \\
E(13,139) & \subset\{1,2\}, & E(127,139)=\varnothing . \\
E(19,139) & \subset\{2\}, & &
\end{array}
$$


Below we give the details of the proof that $E(3,139) \subset\{2,4\}$. These details are fairly representative of the techniques used in establishing the remainder of (3), the proof of which we omit.

Since $a_{3}(3,139)+b(3,139)+3=19<69=1 / 2(139-1)$, (viii) and (ix) of Theorem 1 imply that if $a \in E(3,139)$, then every prime divisor of $a+1$ is less than 69. Since every prime divisor of $\sigma\left(3^{a}\right)$ is at least 139 for $a=30,36,42,46,58,60$, 66, (vi) and (ix) of Theorem 1 imply that if $a \in E(3,139)$, then $(a+1,31 \cdot 37 \cdot 43 \cdot$ $47 \cdot 59 \cdot 61 \cdot 67)=1$. Since $1093\left|\sigma\left(3^{6}\right), 3851\right| \sigma\left(3^{10}\right), 1871\left|\sigma\left(3^{16}\right), 1597\right| \sigma\left(3^{18}\right)$, 28537 $\sigma\left(3^{28}\right)$, (ii) and (ix) of Theorem 1 imply that if $a \in E(3,139)$, then $(a+1,7$. $11 \cdot 17 \cdot 19 \cdot 29)=1$. Let $m_{2}=\sigma\left(3^{52}\right) / 107, \sigma\left(3^{40}\right) / 83$, or $\sigma\left(3^{22}\right) / 47$. Then in each case $m_{2}$ is an integer, every prime divisor of $m_{2}$ is at least 139 , and $a_{3}\left(m_{2}\right)+$ $v_{3}\left(m_{2}-1\right)=1$. Then (vii) and (ix) of Theorem 1 imply that if $a \in E(3,139)$, then $(a+1,23 \cdot 41 \cdot 53)=1$. Since $4 \mid \sigma(3)$, (i) and (ix) of Theorem 1 imply that if $a \in$ $E(3,139)$, then $(a+1,2)=1$.

Hence if $a \in E(3,139)$, then every prime divisor of $a+1$ is found in the set $\{3,5,13\}$. Suppose $n \in P(139), 3^{a} \| n$, and $13 \mid(a+1)$. Then $p_{1}=797161=\sigma\left(3^{12}\right)$ is the largest prime divisor of $n$. Say $p_{1}^{a} 1 \| n$. Now $797161 \notin Q(3,139)$, so Lemma 5 implies $v_{3}\left(\sigma\left(797161^{a}\right)\right) \geqslant a-a_{2}(3,139)=a-10 \geqslant 2$. Then Lemma 2 implies $9 \mid\left(a_{1}+1\right)$. Hence $151\left|\sigma\left(797161^{2}\right)\right| \sigma\left(797161^{a} 1\right) \mid 2 n$, contradicting $n \in P(139)$. Hence if $a \in E(3,139)$, then $(a+1,13)=1$. Since $757\left|\sigma\left(3^{8}\right), 4561\right| \sigma\left(3^{14}\right)$, and 8951| $\sigma\left(3^{24}\right)$, (ii) and (ix) of Theorem 1 imply that if $a \in E(3,139)$, then $9 /(a+1), 15 /(a+1)$, $25 /(a+1)$. We conclude that $E(3,139) \subset\{2,4\}$.

We now use (3) to prove (2). Suppose $n \in P(139)$. Then (3) implies $31 / n$ and $127 / n$. Now, if $19 \mid n$, (3) implies $19^{2} \| n$. But $\sigma\left(19^{2}\right)=3 \cdot 127$ and $127 / n$. Hence $19 \gamma n$. If either $7 \mid n$ or $11 \mid n$, then $7^{2} \| n$ or $11^{2} \| n$, respectively. But $\sigma\left(7^{2}\right)=3 \cdot 19$ and $\sigma\left(11^{2}\right)=7 \cdot 19$, and $19 / n$. Hence $7 / / n$ and $11 / / n$. If $97 / n$, then $97 \| n$. But $\sigma(97)=2 \cdot$ $7^{2}$ and $7 / / n$. Hence $97 / / n$. If $61 \mid n$, then either $61 \| n$ or $61^{2} \| n$. But $\sigma(61)=2 \cdot 31$, $\sigma\left(61^{2}\right)=3 \cdot 13 \cdot 97$, and $31 / n, 97 / \eta n$. Hence $61 / n$. If $13 \mid n$, then $13 \| n$ or $13^{2} \| n$. But $\sigma(13)=2 \cdot 7, \sigma\left(13^{2}\right)=3 \cdot 61$, and $7 / / n, 61 / n$. Hence $13 / / n$. Finally, if $3 \mid n$, then $3^{2} \| n$ or $3^{4} \| n$. But $\sigma\left(3^{2}\right)=13, \sigma\left(3^{4}\right)=11^{2}$, and $11 / n, 13 / n$. Hence $3 / n$.

Summing up, if $n \in P(139)$, then $(n, 3 \cdot 7 \cdot 11 \cdot 13 \cdot 19 \cdot 31 \cdot 61 \cdot 97 \cdot 127)=$ 1. Say the prime factorization of $n$ is $p_{1}^{a_{1}} p_{2}^{a_{2}} \cdots p_{t}^{a} t$ where $p_{1} \geqslant 100129>139>$ $p_{2}>\cdots>p_{t}$. Then

$$
\begin{aligned}
2 & =\frac{\sigma(n)}{n}=\prod_{i=1}^{t} \frac{\sigma\left(p_{i}^{a_{i}}\right)}{p_{i}^{a_{i}}} \\
& =\prod_{i=1}^{t} \frac{p_{i}^{a_{i}+1}-1}{p_{i}^{a_{i}}\left(p_{i}-1\right)}<\prod_{i=1}^{t} \frac{p_{i}}{p_{i}-1} \leqslant \frac{100129}{100128} P<1.9
\end{aligned}
$$

where $P$ is the product of all $p /(p-1)$ as $p$ ranges over all primes less than 139 and not equal to $2,3,7,11,13,19,31,61,97,127$. This contradiction shows that $P(139)$ $=\varnothing ;$ that is, if an opn exists, its second largest prime factor is at least 139 . 
4. An Application. It was noted by Euler [3], that if $n$ is an opn, then in $n$ 's prime factorization, every exponent is even except for one exponent which is $\equiv 1$ $(\bmod 4)$ as is the corresponding prime. We also note that if $p$ is a prime, then $\sigma\left(p^{a}\right) / p^{a}$ is an increasing function of $a$ and $\lim _{a \rightarrow \infty} \sigma\left(p^{a}\right) / p^{a}=p /(p-1)$. We are now in a position to prove:

THEOREM 2. If $n=p_{1}^{a_{1}} p_{2}^{a_{2}} \cdots p_{7}^{a_{7}}$ is an opn where $p_{1}>p_{2}>\cdots>p_{7}$ are primes and $a_{1}, a_{2}, \ldots, a_{7}$ are positive integers, then $p_{7}=3$ and $p_{6}=5$ or 7 .

Proof. As we remarked in Section 1, Sylvester [11] proved that $p_{7}=3$.

Suppose $p_{6} \geqslant 11$. Then $p_{6}=11, p_{5}=13, p_{4}=17$, and $p_{3}=19$. Indeed, if not, using (1) and (2) we have

$$
\begin{aligned}
2 & =\frac{\sigma(n)}{n}=\prod_{i=1}^{7} \frac{\sigma\left(p_{i}^{a_{i}}\right)}{p_{i}^{a_{i}}}<\prod_{i=1}^{7} \frac{p_{i}}{p_{i}-1} \\
& \leqslant \frac{3}{2} \cdot \frac{11}{10} \cdot \frac{13}{12} \cdot \frac{17}{16} \cdot \frac{23}{22} \cdot \frac{139}{138} \cdot \frac{100129}{100128}<2,
\end{aligned}
$$

a contradiction. We next note that $a_{7} \geqslant 4, a_{6} \geqslant 4, a_{5} \geqslant 4$, and $a_{3} \geqslant 4$. Indeed, if $a_{7}=2$, then

$$
\frac{\sigma(n)}{n}<\frac{\sigma\left(3^{2}\right)}{3^{2}} \cdot \frac{11}{10}: \frac{13}{12} \cdot \frac{17}{16} \cdot \frac{19}{18} \cdot \frac{139}{138} \cdot \frac{100129}{100128}<2 .
$$

If $a_{6}=2$, then $7\left|\sigma\left(11^{2}\right)\right| n$. If $a_{5}=1$, then $7|\sigma(13)| 2 n$. If $a_{5}=2$, then $61\left|\sigma\left(13^{2}\right)\right| n$. If $a_{3}=2$, then $127\left|\sigma\left(19^{2}\right)\right| n$.

We now show that $a_{4} \geqslant 4$. First, suppose $a_{4}=2$. Then $p_{2}=307=\sigma\left(17^{2}\right)$. Then

$$
\frac{\sigma(n)}{n}>\frac{\sigma\left(3^{4}\right)}{3^{4}} \cdot \frac{\sigma\left(11^{4}\right)}{11^{4}} \cdot \frac{\sigma\left(13^{4}\right)}{13^{4}} \cdot \frac{\sigma\left(17^{2}\right)}{17^{2}} \cdot \frac{\sigma\left(19^{4}\right)}{19^{4}} \cdot \frac{\sigma\left(307^{2}\right)}{307^{2}}>2,
$$

a contradiction. Next suppose $a_{4}=1$. Then from 1.13 in [9], we have $p_{1} \equiv p_{2} \equiv 1$ $(\bmod 17)$. But $p_{2} \geqslant 139$, so $p_{2} \geqslant 239$. Then since

$$
\frac{\sigma\left(3^{4}\right)}{3^{4}} \cdot \frac{11}{10} \cdot \frac{13}{12} \cdot \frac{\sigma(17)}{17} \cdot \frac{19}{18} \cdot \frac{239}{238} \cdot \frac{100129}{100128}<2,
$$

we have $a_{7} \geqslant 6$. But $1093 \mid \sigma\left(3^{6}\right)$ and $1093 \neq 1(\bmod 17)$, so $a_{7} \geqslant 8$. Now

$$
\frac{\sigma\left(3^{8}\right)}{3^{8}} \cdot \frac{\sigma\left(11^{4}\right)}{11^{4}} \cdot \frac{\sigma\left(13^{4}\right)}{13^{4}} \cdot \frac{\sigma(17)}{17} \cdot \frac{\sigma\left(19^{4}\right)}{19^{4}} \cdot \frac{\sigma\left(647^{2}\right)}{647^{2}}>2 \text {, }
$$

so $p_{2}>647$. Then since $p_{2} \equiv 1(\bmod 17)$, we have $p_{2} \geqslant 919$. Then

$$
\frac{\sigma(n)}{n}<\frac{3}{2} \cdot \frac{11}{10} \cdot \frac{13}{12} \cdot \frac{\sigma(17)}{17} \cdot \frac{19}{18} \cdot \frac{919}{918} \cdot \frac{100129}{100128}<2,
$$

a contradiction. Hence $a_{4} \geqslant 4$.

Also since

$$
\frac{\sigma\left(3^{6}\right)}{3^{6}} \cdot \frac{\sigma\left(11^{4}\right)}{11^{4}} \cdot \frac{\sigma\left(13^{4}\right)}{13^{4}} \cdot \frac{\sigma\left(17^{4}\right)}{17^{4}} \cdot \frac{\sigma\left(19^{4}\right)}{19^{4}}>2,
$$

we have $a_{7}=4$. 
Using the natural generalization of 1.11 from [9] , since $a_{4} \geqslant 4$, we have for either $p=p_{1}$ or $p=p_{2}$ that $p \equiv 1(\bmod 4)$ and $p \equiv-1\left(\bmod 17^{4}\right)$. Then $p \equiv$ $167041(\bmod 334084)$. But 167041 is not prime, so $p>500000$. Now, if $p_{2} \geqslant 569$, we have

$$
\frac{\sigma(n)}{n}<\frac{\sigma\left(3^{4}\right)}{3^{4}} \cdot \frac{11}{10} \cdot \frac{13}{12} \cdot \frac{17}{16} \cdot \frac{19}{18} \cdot \frac{569}{568} \cdot \frac{500001}{500000}<2 .
$$

Hence $p_{2} \leqslant 563$. But then

$$
\frac{\sigma(n)}{n}>\frac{\sigma\left(3^{4}\right)}{3^{4}} \cdot \frac{\sigma\left(11^{4}\right)}{11^{4}} \cdot \frac{\sigma\left(13^{4}\right)}{13^{4}} \cdot \frac{\sigma\left(17^{4}\right)}{17^{4}} \cdot \frac{\sigma\left(19^{4}\right)}{19^{4}} \cdot \frac{\sigma(563)}{563}>2,
$$

a contradiction.

\section{TABLE 1}

\begin{tabular}{rccl}
$p$ & $b(p, 139)$ & $a_{1}(p, 139)$ & \multicolumn{1}{c}{$A_{1}(p, 139)$} \\
3 & 3 & 3 & $5,5^{5}, 17,29,41,53,89,101,113,137$ \\
7 & 1 & 2 & $5^{5}, 13,41,97$ \\
11 & 1 & 1 & 109 \\
13 & 1 & 0 & $\varnothing$ \\
19 & 0 & 1 & 37,113 \\
31 & 0 & 1 & $37^{5}, 61$ \\
61 & 0 & 0 & $\varnothing$ \\
97 & 0 & 0 & $\varnothing$ \\
127 & 0 & 0 & $\varnothing$
\end{tabular}

TABLE 2

\begin{tabular}{rrl}
$p$ & $a_{2}(p, 139)$ & \multicolumn{1}{c}{$A_{2}(p, 139)$} \\
3 & 10 & $7^{2}, 13^{2}, 19^{2}, 37^{2}, 61^{2}, 67^{2}, 79^{2}$ \\
7 & 11 & $11^{2}, 23^{2}, 37^{2}, 67^{2}, 79^{2}, 107^{2}, 137^{2}$ \\
11 & 4 & $3^{4}, 5^{4}$ \\
13 & 4 & $3^{2}, 29^{2}, 61^{2}, 107^{2}$ \\
19 & 3 & $7^{2}, 11^{2}$ \\
31 & 3 & $5^{2}, 67^{2}$ \\
61 & 2 & $13^{2}, 47^{2}$ \\
97 & 1 & $61^{2}$ \\
127 & 2 & $19^{2}, 107^{2}$
\end{tabular}


TABLE 3

\begin{tabular}{rrll}
$p$ & $a_{3}(p, 139)$ & \multicolumn{1}{c}{$A_{3}(p, 139)$} & \multicolumn{1}{c}{$Q(p, 139)$} \\
3 & 13 & $13^{8}, 61^{8}, 67^{8}$ & $1609669,903870199,30152894311$ \\
7 & 12 & $29^{6}$ & 88009573 \\
11 & 8 & $23^{10}, 97^{4}, 103^{4}, 137^{4}$ & $262321,319411,10332211,3937230404603$ \\
13 & 4 & $\varnothing$ & $\varnothing$ \\
19 & 6 & $23^{8}, 47^{8}, 61^{8}$ & $7792003,567332587,903870199$ \\
31 & 4 & $97^{4}$ & 262321 \\
61 & 3 & $131^{4}$ & 973001 \\
97 & 1 & $\varnothing$ & $\varnothing$ \\
127 & 2 & $\varnothing$ & $\varnothing$
\end{tabular}

Department of Mathematics

University of Georgia

Athens, Georgia 30602

1. A. S. BANG, "Taltheoretiske Underspgelser," Tidsskrift Math., v. 5, 1886, pp. 70-80, $130-137$.

2. L. E. DICKSON, "Finiteness of the odd perfect and primitive abundant numbers with $n$ distinct prime factors," Amer. J. Math., v. 35, 1913, pp. 413-422.

3. L. EULER, "Tractatus de numerorum doctrina," Commentationes Arithmeticae Collectae, v. 2, 1849, p. 514 .

4. I. S. GRADŠTEIN, “O ne飞etnych soverక̌ennych đislah,” Mat. Sb., v. 32, 1925, pp. 476510.

5. P. HAGIS, JR. \& W. L. MCDANIEL, "On the largest prime divisor of an odd perfect number," Math. Comp., v. 27, 1973, pp. 955-957. MR 48 \#3855.

6. P. HAGIS, JR. \& W. L. MCDANIEL, "On the largest prime divisor of an odd perfect number. II," Math. Comp., v. 29,1975, pp. 922-924.

7. H.-J. KANOLD, "Folgerungen aus dem Vorkommen einer Gaussschen Primzahl in der Primfaktorenzerlegung einer ungeraden vollkommenen Zahl," J. Reine Angew. Math., v. 186, 1944, pp. 25-29. MR 6, 255.

8. T. NAGELL, Introduction to Number Theory, 2nd ed., Chelsea, New York, 1964. MR 30 \#4714.

9. C. POMERANCE, “Odd perfect numbers are divisible by at least seven distinct primes," Acta Arith., v. 25, 1974, pp. 265-300.

10. N.ROBBINS, The Non-Existence of Odd Perfect Numbers With Less Than Seven Distinct Prime Factors, Doctoral Dissertation, Polytechnic Institute of Brooklyn, June 1972.

11. J. J. SYLVESTER, "Sur une classe spéciale des diviseurs de la somme d'une série géométrique," Comptes Rendus, v. CVI, 1888, pp. 446-450.

12. J. J. SYLVESTER, "Sur les nombres parfaits," Comptes Rendus, v. CVI, 1888, pp. 403-405. 